\title{
An Expert System for Workflow Procedure Optimization
}

\author{
Xiaoping Qiu${ }^{1}$ Xiaobing $\mathrm{Li}^{2}$ Yang $\mathrm{Xu}^{2}$ \\ ${ }^{1}$ College of Logistics, Southwest Jiaotong University, Chengdu610031, P.R.China \\ ${ }^{2}$ Intelligent Control Development Center, Southwest Jiaotong University, Chengdu610031, P.R.China
}

\begin{abstract}
An expert system for workflow procedure optimization is proposed in this paper, in which the optimization method of the workflow procedure is presented with the workflow management system (abbr. WfMS). The running analysis of WfMS is the inputs of expert system, the optimization report and the regulation implementation for WfMS are the outputs of it. The optimization model and the disposal procedure are discussed in detail including the relative data tables and the reasoning algorithm in expert system. All those works make it be a feasible expert system for workflow procedure optimization.
\end{abstract}

Keywords: Procedure optimization, Workflow technology, Expert system

\section{Introduction}

Workflow technology is a new hotspot in the area of computer application after 1990s and is widely applied in many information system including EService, E-business, and so on $[1,2]$. With the development of information technology, more and more intelligent method and technology are adopted into workflow management system (abbr. WfMS)[3]. In the paper [4], the case-base reasoning method is used to support modelling and problem solving with the precedent experiences. In the paper[5], the data mining method is used to extract the workflow critical path. All those research show that the connection of workflow technology and the artificial intelligence becomes closer, even for other suitable technology like the agent technology [6].

As we known, the workflow process can be regulated conveniently in workflow management system for the developing requirements $[7,8,9]$. But why and how we regulate the workflow procedure still are the key issue in this field. Based on our previous works[10, 11], we have discussed the regulation method in paper [12], in which the addition, the modification and the deletion of the activities in workflow procedure are analyzed based on the three basic relationships among the actives.

As to the 'why' problem, we have put forward an universal hierarchical intelligent system for evaluating the status of the workflow procedure and its actives [13] in WfMS. With those works, we can find out which activity needs to deal. The relations between the disposal methods and the evaluation results are complex but it's feasible for the experts. So the expert system is adopted to manage this issue by using the experiences of the relative experts.

So the optimization model of the workflow procedure are shown in Figure 1. The running analysis of WfMS is discussed in [14], which consists of three aspect usually, such as time, cost and material. For convenience, the functions are used to analyze those status. In the following sections, the expert system is described based on the functions in detail.

\section{The optimization circle}

From the optimization model in Figure 1, the optimization circle can be obtained easily, which is shown in Figure 2.

The experiences of the experts are expressed by rule and saved in rule base. In order to learn the experiences of the expert at any time, the interface to manage the rules is given, so is the procedure regulation. Those interface will be weaken when the expert system becomes mature. Obviously, the optimization circle can run again and again for WfMS.

For the expert system, the inputs, the optimization rules and the outputs are the important components. So they are presented in turn as follow based on the database technology for constructing the applied system [15].

\subsection{The inputs}

As the mention above, the analysis and evaluation results are expressed as the functions with para- 


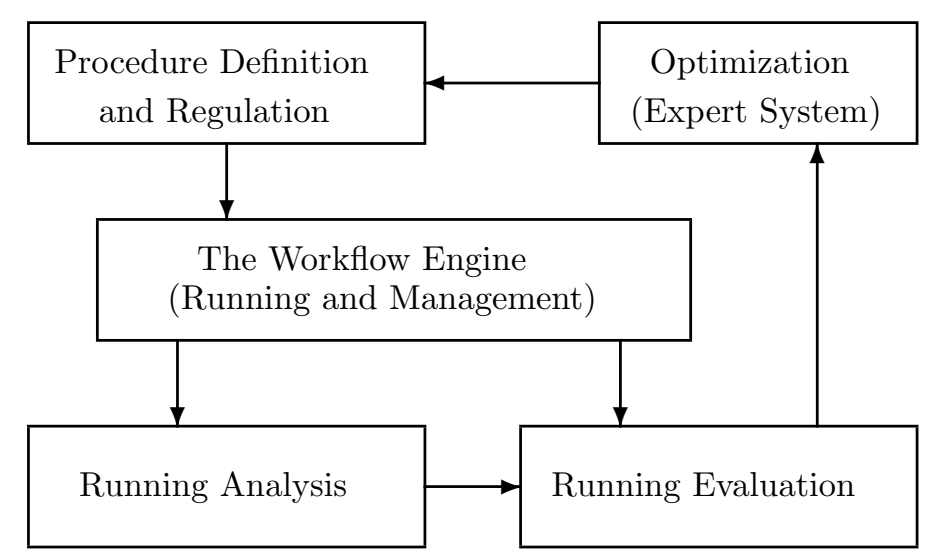

Fig. 1: The optimization model

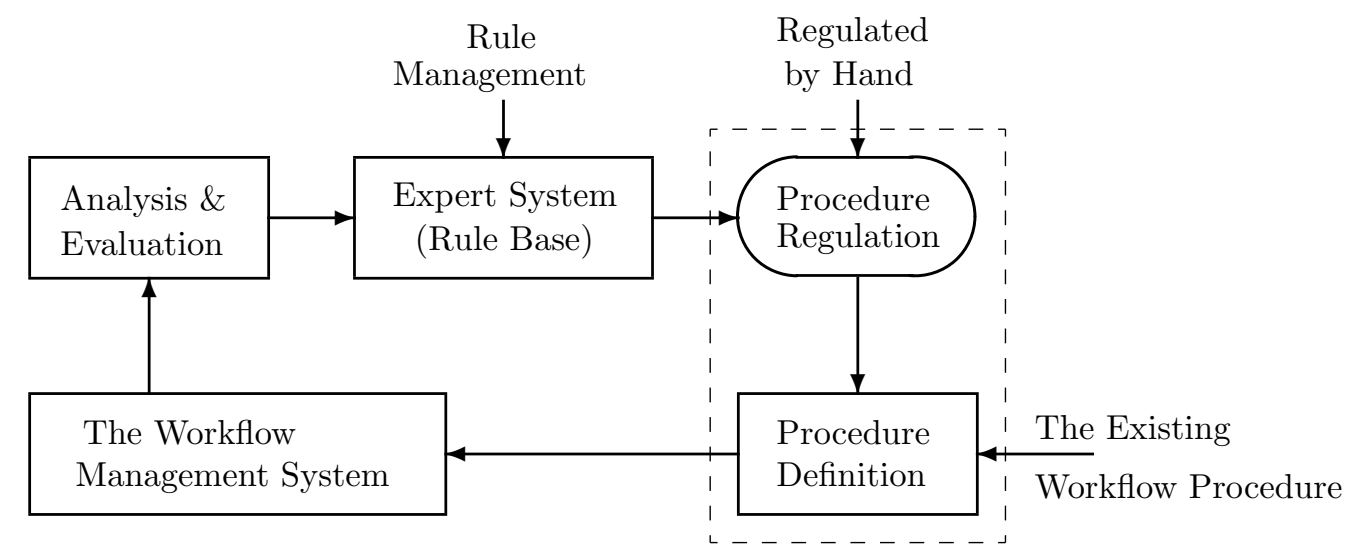

Fig. 2: The optimization circle

meters. The parameters are different for different objects. So the function list table (FLT) is needed to save them, which is shown in Table 1. In FLT, the combination of the first two fields is the primary key (it is same for the following tables).

\begin{tabular}{ccc}
\hline Attribute & Type & Length \\
\hline Function No & VarChar & 3 \\
Function Name & VarChar & 10 \\
Description & VarChar & 50 \\
Function Type & Char & 1 \\
IsSelected & Char & 1 \\
Procedure No & Char & 2 \\
Activity No & Char & 3 \\
\hline
\end{tabular}

Table 1: The Function List Table (FLT)
In Table 1,1) the parameters of those functions are ignored, if necessary, another table is needed; 2) For Computation, some basic ration data is needed, such as labor force; 3 ) there are two function types, one for procedure and the other for its activity; 4) the last fields are used when computing, the procedure no. and the activity no. are from the corresponding tables which save their information.

\subsection{The optimization rules}

The optimization rule table saves the experience of the relative experts and the attributes of the rule are list in Table 2.

In Table 2,1 ) there are two rule types including the advice type and the execution type; 2) there are three operation types including the addition, the modification and the deletion; 3) the precondition of the rule consists of three fields including 'Pre- 


\begin{tabular}{ccc}
\hline Attribute & Type & Length \\
\hline Rule No & VarChar & 2 \\
Rule Name & VarChar & 10 \\
Description & VarChar & 50 \\
Rule Type & Char & 1 \\
PreCondition & Char & 5 \\
PreRelation & Char & 1 \\
PreValue & VarChar & 10 \\
Operation Type & Char & 1 \\
RelativeInfo & VarChar & 30 \\
Priority & Integer & Default \\
IsValid & Char & 1 \\
\hline
\end{tabular}

Table 2: The Optimization Rule Table(ORT)

Condition','PreRelation', and 'Prevalue', in which the 'PreRelation' has three values: ' 0 ' for 'equal', ' 1 ' for 'no less than', and ' 2 ' for 'no greater than'; 4) the conclusion of the rule consists of the fields 'Operation Type' and 'RelativeInfo', the latter field is filled for the necessary information, such as the activity no. to be operated; 4) the field 'Priority' is used to solve the conflict of the rules with the same precondition; 5) the field 'IsValid' is used to control some unusual rules.

\subsection{The outputs}

The expert system runs on the results of the running analysis and evaluation. The main work of the expert system is to infer the proper rule. So the analysis result table (ART) is also used as the outputs of the expert system. The attributes of those results are list in Table 3.

\begin{tabular}{ccc}
\hline Attribute & Type & Length \\
\hline Analysis No & VarChar & 5 \\
Analysis Name & VarChar & 3 \\
Description & VarChar & 12 \\
Analysis Value & VarChar & 10 \\
Analysis Time & DateTime & Default \\
Rule No & VarChar & 3 \\
Use Time & DateTime & Default \\
Result & VarChar & 50 \\
\hline
\end{tabular}

Table 3: The Analysis Result Table (ART)

In Table 3, 1) the analysis no. is composed by the procedure no. and the function no.; 2) the analysis value is used to save the analysis result;
After the comparison between this value and the 'PreValue' based on the 'PreRelation' in ORT, the 'Rule No' field will be filled with that the matched rule; 3 ) the analysis time and the use time are used to record the corresponding operation time for validation, the result to save another necessary information; 4) the history of ART can be saved in another table with the same fields, but the primary key must be the combination of the analysis no. and the analysis time.

\section{The reasoning algorithm}

In practice, the optimization procedure consists of the following steps, such as selecting the analysis object, specifying the analysis functions, executing the analysis procedure and saving the analysis result in ART. Then the expert system will be worked based on ART and the operation will be executed by the executor if necessary based on the reasoning results. The implementation procedure is shown in Figure 3, in which the components of the expert system are illustrated in the dash frame.

In Figure 3, the analyzer, the reasoning machine and the executer are executable programs, they read and write the necessary data automatically and execute the operations based on their judgements.

The reasoning algorithm is the key component in the reasoning machine, by which the reasoning machine can match the proper rule for every analysis result in ART. After this work, the 'Rule no' in ART are filled, then the optimization report can be generated and the executor may be driven for an practical regulation. Here the reasoning algorithm is shown as follows.

1. Let $n$ be the record number in ART, sort ART by "Analysis No. Ascending", $i=1$;

2. Judge " $i>n$ ?", if yes, then go to step 13, otherwise, execute the following statements;

3. Read the "Analysis No." and "Analysis Value" field of the $i$-th in ART and save them into the variables $A_{n}, A_{v}$ respectively, calculate $f_{n}=\operatorname{Right}\left(A_{n}, 2\right)$;

4. Filter the records in ORT by the condition of "PreCondition $=f_{n}$ " and "IsValid $=$ ' 1 '";

5. Let $m$ be the record number in filtered ORT, sort ORT by "Priority Ascending", $j=1$;

6. Judge " $j>m$ ?", if yes, then go to step 11 , otherwise, execute the following statements;

7. Read the "PreRelation", "PreValue" and "Rule No" field of the $j$-th in ORT and save them into the variable $P_{R}, P_{V}$ and $R_{N}$; 


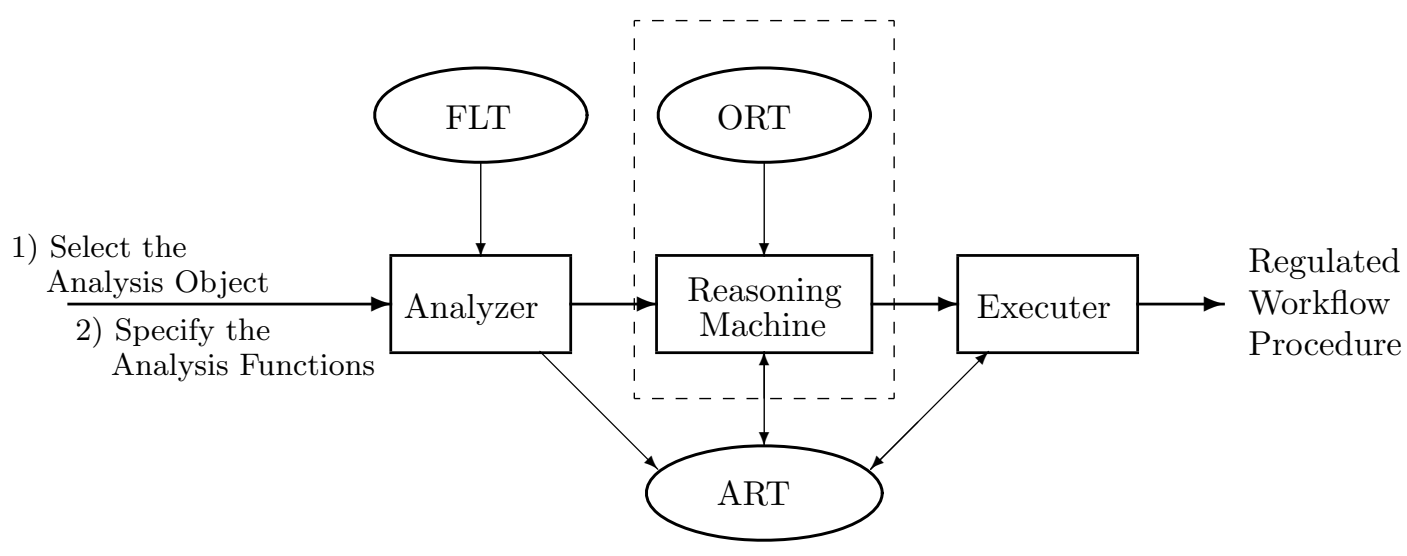

Fig. 3: The regulation implementation procedure of the workflow procedure

8. Let the boolean variable $I$ sMatch $=$ False and execute the following statements:

8.1. If $P_{R}={ }^{\prime} 0$ ' and $A_{V}=P_{V}$, then IsMatch $=$ True;

8.2. If $P_{R}={ }^{\prime} 1$ ' and $A_{V} \geq P_{V}$, then IsMatch $=$ True;

8.3. If $P_{R}=$ '2' and $A_{V} \leq P_{V}$, then IsMatch $=$ True;

9. Judge "IsMatch $=$ True ?", If yes, then write the value of $R_{N}$ into the 'Rule No' field in the $i$-th record, Let $j=m$;

10. $j=j+1$, go to step 6 ;

11. Prompt " No proper optimization rule for the $i$-th analysis result.";

12. $i=i+1$, go to step 2;

13. Prompt "The reasoning algorithm is over.";

14. The algorithm terminates.

Obviously, the computational complexity of the reasoning algorithm is $O\left(n^{2}\right)$ because one nested circle is used. Based on this work, the optimization report or the regulation execution can be realized subsequently.

\section{Conclusions}

This paper gives an expert system for workflow procedure optimization based on the database technology. It is important for workflow technology because the idea of the self-optimization of WfMS is unveiled to us. More intelligent theories and methods will step into WfMS for more convenience.

\section{Acknowledgements}

This research is supported by two National Natural Science Fund of China (Grant No.: 60474022 \& 70502025) and the specialized Research Fund for the Doctoral Program of Higher Education of China under Grant No. 20060613007.

\section{References}

[1] Workflow Management Coalition. The workflow reference model (WFMC-TC00-1003). Brussels, 1995.

[2] Y. Fan. Fundamentals of workflow management technology. TUP \& Springer, Beijing, 2001(in Chinese).

[3] M.D.R. Moreno, P. Kearney. Integrating AI planning techniques with workflow management system. Knowledge-Based System. 2002, 15(5): 285-191

[4] D.S. Kaster, C.B. Medeiros, H.V. Rocha. Supporting modelling and problem solving from precedent experiences: the role of workflows and case-based reasoning. Environmental Modelling and Software. 2005, 20(6): 689-704

[5] J.H. Son, J.S. Kim, M.H. Kim. Extracting the workflow critical path from the extended wellformed workflow schema. Journal of Computer and System Sciences. 2005, 70(1): 86-106

[6] M. Wang, H. Wang, D. Xu. The design of intelligent workflow monitoring with agent technology. Knowledge-Based Systems. 2005, 18(6), October, 2005, 257-266

[7] D.E. Mahling, N. Craven, W.B. Croft. From office automation to intelligent workflow sys- 
tems. IEEE Intelligent system, 1995, 10(3), pp. $41-47$

[8] C. Wenzel. Integrating information extraction into workflow management systems. Proc. 9th International Workshop on Database and Expert Systems Applications, pp. 137-142, Aug. 1998.

[9] M. Shikida, C. Kadowaki, S. Kunifuji. Towards a real-world oriented workflow system - based on practical experiments for three years. Proc. 3rd International Conference Knowledge-Based Intelligent Information Engineering Systems, pp. 46-49, Dec. 1999.

[10] X. Qiu, J. Zheng, Y. Tang, Y. Xu. Executive Validation Analysis of Workflow Process. Proc. $5^{\text {th }}$ World Congress on Intelligent Control and Automation, Vol.3, Jun. 2004, pp: $2702-2705$

[11] X. Qiu, Y. Xu, X. Liu, G. Zhang, Workflow Model of Intelligent Office Information System, Proc. 2004 IEEE Interna- tional Conference on Systems, Man and $\mathrm{Cy}$ bernetics, Vol.1, Oct. 2004, pp: 485-489

[12] X. Qiu, Y. Wang, H. Li, D. Meng. An Intelligent Adjustive Method for Workflow Management System, Proc. $8^{\text {th }}$ Joint Conference on Information Sciences, Salt Lake, July 21-26, 2005, pp: 1602-1605

[13] X. Qiu, Y. Xu, M. Jian, H. Li. The Hierarchical Fuzzy Evaluation System and Its Application, Lecture Notes on Artificial Intelligent. Mar. 2006, Vol.3930, pp: 407-416.

[14] X. Qiu. Study on Intelligent Office Information System Based on Workflow Technology. PostDoctoral Report of Southwest Jiaotong University, October 2006

[15] L. M. Barone, S. Cau, F. Cavallari, S. Costantini, I. Dafinei, M. Diemoz, et. al. REDACLE: A flexible database for traceability and workflow management for detector construction. Computer Physics Communications. 2006, 174(11): 869-875 